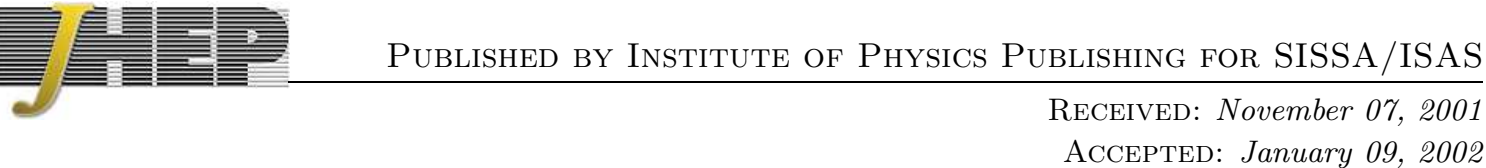

ACCEPTED: January 09, 2002

\title{
Finite Chern-Simons matrix model - algebraic approach
}

\author{
Larisa Jonke and Stjepan Meljanac \\ Theoretical Physics Division, Rudjer Bošković Institute \\ P.O. Box 180, HR-10002 Zagreb, Croatia \\ E-mail: larisa@thphys.irb.hy, meljanac@thphys.irb.hr
}

\begin{abstract}
We analyze the algebra of observables and the physical Fock space of the finite Chern-Simons matrix model. We observe that the minimal algebra of observables acting on that Fock space is identical to that of the Calogero model. Our main result is the identification of the states in the l-th tower of the Chern-Simons matrix model Fock space and the states of the Calogero model with the interaction parameter $\nu=l+1$. We describe quasiparticle and quasihole states in the both models in terms of Schur functions, and discuss some non-trivial consequences of our algebraic approach.
\end{abstract}

KeYwords: Chern-Simons Theories, Non-Commutative Geometry, Matrix Models, Integrable Field Theories. 


\section{Contents}

1. Introduction 1

2. CS matrix model 2

2.1 Introduction - the physical Fock space 2

2.2 Algebraic structure of the CS matrix model

2.3 Relation to the Calogero model 5

3. Quasiparticles and quasiholes 7

4. Outlook and discussion 8

A. Calogero model 10

\section{Introduction}

It is well known that the canonical quantization of the system of particles in a strong magnetic field gives a natural realization of non-commutative space. One can speculate whether it is possible to describe a real physical system - Quantum Hall fluid - using quantum field theory on non-commutative plane. It was conjectured [1] that the Laughlin state of electrons at a filling fraction ${ }^{1} 1 / k$ was described by the non-commutative version of the $\mathrm{U}(1)$ Chern-Simons theory at level $k$. The fields in that theory were infinite matrices corresponding to an infinite number of electrons on infinite plane. Later, Polychronakos [2] proposed a regularized version of the same model that could describe a finite number of electrons localized on a plane. The complete minimal basis of exact wavefunctions for the theory at an arbitrary level $k$ and rank $N$ was given in ref. [3], and the coherent-state representation was analyzed in ref. [4]. Using the properties of the energy eigenvalues of the Calogero model [5], an orthogonal basis for the Chern-Simons (CS) matrix model was identified [6].

The relation between the Calogero model and Quantum Hall (QH) physics was investigated using the algebraic approach [7, 8] and the collective-field theory [9]. In ref. [7] it was conjectured and then proved in ref. [8] that one could map anyons in the lowest Landau level into the Calogero model, using the complex representation of the $S_{N}$-extended Heisenberg algebra underlying the Calogero model. On the other hand, it was shown in ref. [9] that the correlation functions of the QH edge state and the Calogero model were related for the integer interaction parameter $\nu$. Also, the relation between the Calogero model and the matrix

\footnotetext{
${ }^{1}$ Actually, it was shown by Polychronakos that owing to the quantum effects the corresponding filling fraction is $1 /(k+1)$.
} 
model was established [10]. Finally, an interesting link between non-commutative CS theory and QH fluid was provided using branes in massive type-IIA string theory [1]]. Taking into consideration all these relations between the Calogero model, the matrix model and $\mathrm{QH}$ physics, one hopes that this intricate network of connections between the apparently different physical systems will provide useful insight into the underlying structure.

In this letter we analyze in detail the physical Fock space of the finite CS matrix model. We observe that the minimal algebra of observables acting on that Fock space is identical to that of the Calogero model, but we stress that the complete algebraic structures are different. Our main result is the identification of the states in the l-th tower of the CS matrix model Fock space and the states of the Calogero model with the interaction parameter $\nu=l+1$. We discuss some mathematical and physical consequences of this identification. Specialy, we describe quasiparticle and quasihole excitations in the CS matrix model, and using the identification, the corresponding excitations in the Calogero model. We also discuss a possible extension of the physical Fock space to include particles with the fractional statistics. To make this paper self-contained, we add an appendix with relevant expressions and results in the Calogero model [12, 13, 14].

\section{CS matrix model}

\subsection{Introduction - the physical Fock space}

Let us start from the action proposed in ref. [2]:

$$
S=\int d t \frac{B}{2} \operatorname{Tr}\left\{\varepsilon_{a b}\left(\dot{X}_{a}+i\left[A_{0}, X_{a}\right]\right) X_{b}+2 \theta A_{0}-\omega X_{a}^{2}\right\}+\Psi^{\dagger}\left(i \dot{\Psi}-A_{0} \Psi\right) .
$$

Here, $A_{0}$ and $X_{a}, a=1,2$, are $N \times N$ hermitean matrices and $\Psi$ is a complex $N$-vector. The eigenvalues of the matrices $X_{a}$ represent the coordinates of electrons, $A_{0}$ is a gauge field, and $\Psi$ acts like a boundary field. We choose the gauge $A_{0}=0$ and impose the equation of motion for $A_{0}$ as a constraint:

$$
-i B\left[X_{1}, X_{2}\right]+\Psi \Psi^{\dagger}=B \theta
$$

The trace part of eq. (2.2) gives

$$
\Psi^{\dagger} \Psi=N B \theta
$$

Notice that the commutators have so far been classical matrix commutators. After quantization, the matrix elements of $X_{a}$ and the components of $\Psi$ become operators satisfying the following commutation relations:

$$
\begin{aligned}
{\left[\Psi_{i}, \Psi_{j}^{\dagger}\right] } & =\delta_{i j}, \\
{\left[\left(X_{1}\right)_{i j},\left(X_{2}\right)_{k l}\right] } & =\frac{i}{B} \delta_{i l} \delta_{j k} .
\end{aligned}
$$

It is convenient to introduce the operator $A=\sqrt{B / 2}\left(X_{1}+i X_{2}\right)$ and its hermitean conjugate $A^{\dagger}$ obeying the following commutation relations:

$$
\left[A_{i j}, A_{k l}^{\dagger}\right]=\delta_{i l} \delta_{j k}, \quad\left[A_{i j}, A_{k l}\right]=\left[A_{i j}^{\dagger}, A_{k l}^{\dagger}\right]=0 .
$$


Then, one can write the hamiltonian of the model at hand as

$$
H=\omega\left(\frac{N^{2}}{2}+\operatorname{Tr}\left(A^{\dagger} A\right)\right)=\omega\left(\frac{N^{2}}{2}+\mathcal{N}_{A}\right)
$$

$\mathcal{N}_{A}$ being the total number operator associated with $A$ 's. Upon quantization, the constraints (2.2) become the generators of unitary transformations of both $X_{a}$ and $\Psi$. The trace part (2.3) demands that (the l.h.s. being the number operator for $\Psi$ 's) $B \theta \equiv l$ be quantized to an integer. The traceless part of the constraint (2.2) demands that the wavefunction be invariant under $\mathrm{SU}(N)$ transformations, under which $A$ transforms in the adjoint $^{2}$ and $\Psi$ in the fundamental representation.

Energy eigenstates will be $\mathrm{SU}(N)$ singlets; generally, some linear combinations of terms with at least $l N(N-1) / 2 A^{\dagger}$ operators and $N l \Psi^{\dagger}$ fields. Explicit expressions for the wavefunctions were written in ref. [3]:

$$
|\Phi\rangle=\prod_{j=1}^{N}\left(\operatorname{Tr} A^{\dagger j}\right)^{c_{j}} C^{\dagger l}|0\rangle,
$$

where

$$
C^{\dagger} \equiv \varepsilon^{i_{1} \cdots i_{N}} \Psi_{i_{1}}^{\dagger}\left(\Psi^{\dagger} A^{\dagger}\right)_{i_{2}} \cdots\left(\Psi^{\dagger} A^{\dagger N-1}\right)_{i_{N}},
$$

and $A_{i j}|0\rangle=\Psi_{i}|0\rangle=0$.

The system contains $N^{2}+N$ oscillators coupled by $N^{2}-1$ constraint equations in the traceless part of eq. (2.2). Effectively, we can describe the system wih $N+1$ independent oscillators. Therefore, the physical Fock space that consists of all $\mathrm{SU}(N)$-invariant states can be spanned by $N+1$ algebraically independent operators: $B_{n}^{\dagger} \equiv \operatorname{Tr} A^{\dagger n}$ with $n=$ $1,2, \ldots, N$, and $C^{\dagger}$. The operators $B_{k}^{\dagger}$ for $k>N$ can be expressed as a homogeneous polynomial of total order $\mathrm{k}$ in $\left\{B_{1}^{\dagger}, \ldots, B_{N}^{\dagger}\right\}$, with constant coefficients which are common to all operators $A^{\dagger}$ [15]. Since

$$
\operatorname{Tr} A^{k} C^{\dagger l}|0\rangle \equiv B_{k} C^{\dagger l}|0\rangle=0, \quad \forall k, \forall l,
$$

the state $C^{\dagger l}|0\rangle \equiv|0, l\rangle$ can be interpreted as a ground state - vacuum with respect to all operators $B_{k}$. Note that the vacuum is not normalized to one, i.e. $\langle 0, l \mid 0, l\rangle \neq 1$. The whole physical Fock space can be decomposed into towers (modules) built on the ground states with different $l$ :

$$
F_{\text {phys }}^{\mathrm{CS}}=\sum_{l=0}^{\infty} F_{\text {phys }}^{\mathrm{CS}}(l)=\sum_{l=0}^{\infty}\left\{\prod B_{k}^{\dagger n_{k}}|0, l\rangle\right\} .
$$

Clearly, the states from different towers are mutually orthogonal.

\footnotetext{
${ }^{2}$ Note that as $A$ transforms in the reducible representation $\left(N^{2}-1\right)+1$, with the singlet $B_{1} \equiv \operatorname{Tr} A$, one can introduce a pure adjoint representation as $\bar{A}_{i j}=A_{i j}-\delta_{i j} B_{1} / N$. This slightly modifies the commutator (2.5), and completely decouples $B_{1}$ from the Fock space. Physically, this correspond to the separation of the centre-of-mass coordinate as it has been done for the Calogero model in ref. [12]. For the sake of simplicity, this will not be done here.
} 
The action of the hamiltonian (2.6) on the states in the physical Fock space is

$$
H \prod B_{k}^{\dagger n_{k}}|0, l\rangle=\omega\left[\frac{N^{2}}{2}+\sum_{k} k n_{k}+l\left(\begin{array}{c}
N \\
2
\end{array}\right)\right] \prod B_{k}^{\dagger n_{k}}|0, l\rangle,
$$

and the ground-state energy is $E_{0}(l)=\omega\left[N^{2}+l N(N-1)\right] / 2$. Comparing this result with the known one for the Calogero model (see eq. (A.5) in appendix A) we see that the spectra of the two models are identical provided $\nu=l+1$. This has already been noticed in refs. [6, 10]. However, from the identification of spectra it only follows

$$
\left(\prod B_{i}^{\dagger n_{i}}\right)^{\mathcal{N}}|0, l\rangle=\sum\left(\prod B_{k}^{\dagger n_{k}}\right)^{\mathcal{N}}|0\rangle_{l+1},
$$

where the r.h.s. of this relation is, in general, a sum of different terms of total order $\mathcal{N}$ in the observables of the Calogero model. We use the same letter to denote observables in both models. In CS matrix model, $B_{n}=\operatorname{Tr} A^{n}$ and in the Calogero model, $B_{n}=\sum_{i} a_{i}^{n}$, but from the context it should be clear what $B_{n}$ represents. The vacuum in the CS matrix model is denoted as $|0, l\rangle$ and the vacuum in the Calogero model is denoted as $|0\rangle_{\nu}$ (see appendix A).

\subsection{Algebraic structure of the CS matrix model}

Using $\left[A_{i j}, B_{n}^{\dagger}\right]=n\left(A^{\dagger n-1}\right)_{i j}$, we find a general expression for the commutators between observables:

$$
\left[B_{m}, B_{n}^{\dagger}\right]=n \sum_{r=0}^{m-1} \operatorname{Tr}\left(A^{r} A^{\dagger n-1} A^{m-r-1}\right)=m \sum_{s=0}^{n-1} \operatorname{Tr}\left(A^{\dagger s} A^{m-1} A^{\dagger n-s-1}\right) .
$$

One can normally order the r.h.s. of eq. (2.11) using the recurrent relation

$$
\operatorname{Tr}\left(A^{r} A^{\dagger n-1} A^{m-1}\right)=\operatorname{Tr}\left(A^{r-1} A^{\dagger n-1} A^{m}\right)+\sum_{s=0}^{n-2} \operatorname{Tr}\left(A^{r-1} A^{\dagger s}\right) \operatorname{Tr}\left(A^{\dagger n-s-2} A^{m-r-1}\right) .
$$

With the formal mapping $\operatorname{Tr}\left(A^{r} A^{\dagger s} A^{k}\right) \rightarrow \sum_{i} a_{i}^{r} a_{i}^{\dagger s} a_{i}^{k}$, the relation (2.11) is identical to eq. (A.6) for the Calogero model. Also, the recurrent relation (2.12) has its counterpart in the Calogero model with $\nu=1$, with the same formal mapping. In order to close the algebra (2.11), one should include observables of the type $B_{m, n}^{\alpha}=\operatorname{Tr}\left(A^{\dagger i_{1}} A^{i_{2}} A^{\dagger i_{3}} \cdots\right)$, where $m$ is the number of $A^{\dagger}$ 's and $n$ is the number of $A$ 's, in the trace. This algebra $\mathcal{B}_{N}^{\mathrm{CS}}$ is a polynomial generalization of the Lie algebra. We stress that this algebra is larger than the corresponding $\mathcal{B}_{N}^{\text {Cal }}$ algebra in the Calogero model described in detail in ref. [12, since in the CS matrix model we have more than one invariant of order $(m, n)$, owing to matrix multiplication. In other words, in the Calogero model there are more algebraic relations connecting the trace invariants for fixed $N$.

In this paper we restrict ourselves to the minimal algebra including only observables of the type $B_{n}$ and $B_{n}^{\dagger}$, defined with the following relations (including corresponding hermitean conjugate relations):

$$
\left[B_{i_{1}},\left[B_{i_{2}},\left[\ldots,\left[B_{i_{n}}, B_{n}^{\dagger}\right] \ldots\right]\right]=n ! \prod_{\alpha=1}^{n} i_{\alpha} B_{I-n},\right.
$$


where $I=\sum_{\alpha=1}^{n} i_{\alpha}$ and $i_{1}, \ldots, i_{n}, n=1,2, \ldots, N$. These relations can be viewed as a generalization of triple operator algebras defining para-Bose and para-Fermi statistics [16]. The identical successive commutators relations (2.13) holds for the observables acting on

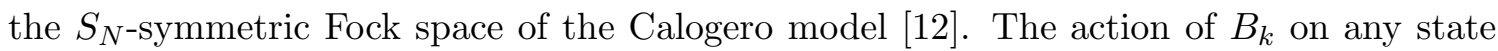
in the Fock space is of the form:

$$
B_{k} \prod_{i} B_{i}^{\dagger n_{i}}|0, l\rangle=\sum\left(\prod B_{j}^{\dagger n_{j}}\right)^{\mathcal{N}-k}|0, l\rangle, \quad \mathcal{N}=\sum_{i} i n_{i} \geq k
$$

In order to calculate the precise form of the r.h.s. of eq. (2.14) we apply the hermitean conjugate relation eq. (2.13) on the l.h.s. of eq. (2.14) shifting the operator $B_{k}$ to the right, at least for one place. We repeat this iteratively as long as the number of $B^{\dagger}$ 's on the right from $B_{k}$ is larger or equal to the index $k$. For $k>\sum n_{i}$, one directly calculates a finite set of relations from (2.5), the so-called generalized vacuum conditions. We show that the minimal set of generalized vacuum conditions needed to completely define the representation of the algebra (2.13) on the Fock space is

$$
\begin{aligned}
B_{2} B_{2}^{\dagger}|0, l\rangle & =2 N(N+l N-l)|0, l\rangle, \\
B_{3} B_{3}^{\dagger}|0, l\rangle & =3 N\left[N^{2}+1+l(N-1)(2 N-1)+l^{2}(N-1)(N-2)\right]|0, l\rangle=y|0, l\rangle, \\
B_{3} B_{3}^{\dagger 2}|0, l\rangle & =54\left\{(l+1) B_{1}^{\dagger} B_{2}^{\dagger}+[N+(N-2) l+y / 27] B_{3}^{\dagger}\right\}|0, l\rangle .
\end{aligned}
$$

Namely, the operators $B_{2}, B_{3}$ and hermitean conjugates play a distinguished role in the algebra, since all other operators $B_{n}, B_{n}^{\dagger}$ for $n \geq 4$ can be expressed as successive commutators (2.13), using only $B_{2}, B_{3}$ and their hermitean conjugates. Therefore, one can derive all other generalized vacuum conditions using (2.13) and (2.15).

The relations (2.13) and the generalized vacuum conditions (2.15) represent the minimal algebraic structure defining the complete physical Fock space representation. Using these relations one can calculate the action of $B_{n}$ on any state in the physical Fock space, and calculate all matrix elements (scalar product) of the form $\left\langle 0, l\left|\left(\prod B_{i}^{n_{i}}\right)\left(\prod B_{j}^{\dagger n_{j}}\right)\right| 0, l\right\rangle$, up to the norm of the vacuum.

\subsection{Relation to the Calogero model}

Our main observation is that the generalized vacuum conditions for the CS matrix model (2.15) and for the Calogero model (A.7) coincide for $\nu=l+1$. This follows entirely from the common algebraic structure (2.13) and the structure of the vacuum conditions. As we have already said, the algebraic relations 2.13) and the generalized vacuum conditions uniquely determine the action of observables on the Fock space, so we conclude that

$$
B_{k}\left(\prod B_{i}^{\dagger n_{i}}\right)|0, l\rangle=B_{k}\left(\prod B_{i}^{\dagger n_{i}}\right)|0\rangle_{l+1},
$$

and that all scalar products in $F_{\text {phys }}^{\mathrm{CS}}$ can be identified with the corresponding matrix elements in the Fock space of the Calogero model:

$$
\left\langle 0, l\left|\left(\prod B_{i}^{n_{i}}\right)\left(\prod B_{j}^{\dagger n_{j}}\right)\right| 0, l\right\rangle={ }_{l+1}\left\langle 0\left|\left(\prod B_{i}^{n_{i}}\right)\left(\prod B_{j}^{\dagger n_{j}}\right)\right| 0\right\rangle_{l+1} .
$$


Note that the vacuum is unique in both models, so we set $|0, l\rangle=|0\rangle_{l+1}$, up to the norm. Now we can identify all states in $F_{\text {phys }}^{\mathrm{CS}}(l)$ with the corresponding states in $F_{\text {symm }}^{\mathrm{Cal}}(l+1)$ as

$$
\left(\prod B_{k}^{\dagger n_{k}}\right)|0, l\rangle=\left(\prod B_{k}^{\dagger n_{k}}\right)|0\rangle_{l+1} .
$$

One can prove these results in different ways. For example, we can restrict ourselves to the subspace of the Fock space generated by $\left\{B_{1}^{\dagger}, B_{2}^{\dagger}, B_{3}^{\dagger}\right\}$ and prove the relations (2.16), (2.17) and (2.18) in this subspace, by straightforward calculation and by induction. Then, using the algebraic relation (2.13) we simply generate the rest of the needed results on the full (physical) Fock space. The other way to obtain our result is to notice that from the equivalence of spectra it follows $\nu=l+1$. Also, from the fact that the algebraic relations (2.13) hold for both models we know that the states in the Calogero model and the CS matrix model are identical, but we have no information on the relation between $l$ and $\nu$. The combination of these two results leads again to the identification of the states in $F_{\text {phys }}^{\mathrm{CS}}(l)$ and $F_{\mathrm{symm}}^{\mathrm{Cal}}(l+1)$, eq. (2.18). We point out that our algebraic proof of eqs. (2.16), (2.17) and (2.18) relies only on the identical algebra (2.13) and the identical minimal set of generalized vacuum conditions for both models, and not on the structure of their hamiltonians.

This identification of the states in the CS matrix model with the corresponding states in the Calogero model is non trivial, from both mathematical and physical point of view. An important consequence of our analysis is that the operators $a_{i}$, defined in eq. (A.3), can be interpreted as elements belonging to the spectrum of the matrix $A$. Namely, they are solutions of the polynomial equation $\operatorname{det}\left|A-a \cdot \mathbb{1}_{N \times N}\right|=0$, i.e. $\sum_{k} \alpha_{k} a^{k}=0$, where $\alpha_{k}$ are operators commuting among themselves and with $a$ 's. Let us assume that there are $N$ different, mutually commuting solutions $a_{1}, \ldots, a_{N}$; then $\operatorname{Tr} A^{n}=\sum a_{i}^{n}$. This pure algebraic relation should be applied to the ground states, according to our results:

$$
\operatorname{Tr} A^{n}|0, l\rangle=\sum_{i} a_{i}^{n}|0\rangle_{l+1}=0, \quad \text { and } \quad \prod\left(\operatorname{Tr} A^{\dagger k}\right)^{n_{k}}|0, l\rangle=\prod\left(\sum_{i} a_{i}^{\dagger k}\right)^{n_{k}}|0\rangle_{l+1} .
$$

Therefore, we conclude that, for consistency reasons, the operators $a_{i}, a_{j}^{\dagger}$ have to satisfy $\left[a_{i}, a_{j}^{\dagger}\right]=-(l+1) K_{i j}, i \neq j$.

Also, as a consequence of this identification, we generate an infinite number of nontrivial identities in the following way. Using the relations valid for $m \leq n$

$$
\begin{aligned}
& {\left[B_{m}, B_{n}^{\dagger}\right]^{\mathrm{CS}}=\sum_{l=1}^{m} c_{l m n}(1) \operatorname{Tr}\left(A^{\dagger n-l} A^{m-l}\right),} \\
& {\left[B_{m}, B_{n}^{\dagger}\right]^{\mathrm{Cal}}=\sum_{l=1}^{m} c_{l m n}(\nu) \sum_{i=1}^{N} a_{i}^{\dagger n-l} a_{i}^{m-l},}
\end{aligned}
$$

where $c_{l m n}(\nu)$ are coefficients depending on all indices and $\nu$, we find an infinite number of identities:

$$
\begin{aligned}
{\left[B_{m}, B_{n}^{\dagger}\right]^{\mathrm{CS}}|0, l\rangle } & =\left[B_{m}, B_{n}^{\dagger}\right]^{\mathrm{Cal}}|0\rangle_{l+1}, \\
\sum_{k=1}^{m} c_{k m n}(1) \operatorname{Tr}\left(A^{\dagger n-k} A^{m-k}\right)|0, l\rangle & =c_{m m n}(l+1) B_{n-m}^{\dagger}|0\rangle_{l+1} .
\end{aligned}
$$


For example, from $\left[B_{2}, B_{n+2}^{\dagger}\right], n>0$ :

$$
\sum_{k=0}^{N-2}(N-k-1)(-)^{k} S_{n-k, 1^{k}}=(N-1) m_{(n)}+\sum_{r=1}^{[n / 2]} m_{(n-r, r)},
$$

where $S_{\lambda}$ is Schur function and $m_{\lambda}$ is symmetric monomial function, see ref. [17].

\section{Quasiparticles and quasiholes}

The low-lying excitations in the CS matrix model can be described in terms of quasiparticles and quasiholes [2]. We can identify these states in a way analogous to that for particles and holes of a Fermi sea. One quasiparticle obtained by exciting a "particle" at Fermi level by energy amount $\Delta E=n \omega$ is

$$
\pi^{\dagger}(n)|0, l\rangle=C^{\dagger(l-1)} \times \varepsilon^{i_{1} \cdots i_{N}} \Psi_{i_{1}}^{\dagger} \cdots\left(\Psi^{\dagger} A^{\dagger N-2}\right)_{i_{N-1}}\left(\Psi^{\dagger} A^{\dagger N-1+n}\right)_{i_{N}}|0\rangle .
$$

One quasihole excitation is obtained by creating a gap inside the QH droplet with energy increase $\Delta E=(N-k) \omega$

$$
\chi^{\dagger}(k)|0, l\rangle=C^{\dagger(l-1)} \times \varepsilon^{i_{1} \cdots i_{N}} \Psi_{i_{1}}^{\dagger} \cdots\left(\Psi^{\dagger} A^{\dagger k-1}\right)_{i_{k}}\left(\Psi^{\dagger} A^{\dagger k+1}\right)_{i_{k+1}} \cdots\left(\Psi^{\dagger} A^{\dagger N}\right)_{i_{N}}|0\rangle .
$$

Observe that $\chi^{\dagger}(N-1)=\pi^{\dagger}(1)$. To see that the charge, i.e. the particle number of a quasihole is quantized as $1 / l$, we simply observe that removing one particle (removing $\left.\left(\Psi^{\dagger} A^{\dagger k}\right)^{l}\right)$ from the ground state is equivalent to creation of $l$ quasiholes.

$$
\begin{aligned}
P^{\dagger}(N) P(k)|0, l\rangle & =\chi^{\dagger l}(k)|0, l\rangle \\
& =\left[C^{\dagger(l-1)} \times \varepsilon^{i_{1} \cdots i_{N}} \Psi_{i_{1}}^{\dagger} \cdots\left(\Psi^{\dagger} A^{\dagger k-1}\right)_{i_{k}}\left(\Psi^{\dagger} A^{\dagger k+1}\right)_{i_{k+1}} \cdots\left(\Psi^{\dagger} A^{\dagger N}\right)_{i_{N}}\right]^{l}|0\rangle .
\end{aligned}
$$

We wish to write the expressions (3.1) and (3.2) in our basis of the physical Fock space, so we introduce the following functions:

$$
\begin{aligned}
\sigma_{n}\left(B_{1}^{\dagger}, \ldots, B_{N}^{\dagger}\right) & =\frac{\varepsilon^{i_{1} \cdots i_{N}} \Psi_{i_{1}}^{\dagger} \cdots\left(\Psi^{\dagger} A^{\dagger N-2}\right)_{i_{N-1}}\left(\Psi^{\dagger} A^{\dagger N-1+n}\right)_{i_{N}}}{C^{\dagger}} \\
\sigma_{1^{N-k}}\left(B_{1}^{\dagger}, \ldots, B_{N}^{\dagger}\right) & =\frac{\varepsilon^{i_{1} \cdots i_{N}} \Psi_{i_{1}}^{\dagger} \cdots\left(\Psi^{\dagger} A^{\dagger k-1}\right)_{i_{k}}\left(\Psi^{\dagger} A^{\dagger k+1}\right)_{i_{k+1}} \cdots\left(\Psi^{\dagger} A^{\dagger N}\right)_{i_{N}}}{C^{\dagger}} .
\end{aligned}
$$

Now, quasiparticle and quasihole excitations are written as $\pi^{\dagger}(n)|0, l\rangle=\sigma_{n}\left(B_{1}^{\dagger}, \ldots, B_{N}^{\dagger}\right)$ $|0, l\rangle$ and $\chi^{\dagger}(k)|0, l\rangle=\sigma_{1^{N-k}}\left(B_{1}^{\dagger}, \ldots, B_{N}^{\dagger}\right)|0, l\rangle$, respectively. In order to precisely determine the functions $\sigma_{\lambda}$, we use the mapping from the CS matrix model to the Calogero model and the fact that the operators $a_{i}^{\dagger}$, defined in eq. (A.3), belong to the spectrum of the matrix $A^{\dagger}$. From the definition of Schur functions $S_{\lambda}$, and using the notation of ref. [17], we have

$$
\begin{aligned}
\sigma_{n}\left(B_{1}^{\dagger}, \ldots, B_{N}^{\dagger}\right)|0, l\rangle & =S_{n}\left(a_{1}^{\dagger}, \ldots, a_{N}^{\dagger}\right)|0\rangle_{l+1}=\sum_{|\lambda|=n} z_{\lambda}^{-1} B_{\lambda_{1}}^{\dagger} \cdots B_{\lambda_{N}}^{\dagger}|0\rangle_{l+1}, \\
\sigma_{1^{N-k}}\left(B_{1}^{\dagger}, \ldots, B_{N}^{\dagger}\right)|0, l\rangle & =S_{1^{N-k}}\left(a_{1}^{\dagger}, \ldots, a_{N}^{\dagger}\right)|0\rangle_{l+1}=\sum_{|\lambda|=N-k} \varepsilon_{\lambda} z_{\lambda}^{-1} B_{\lambda_{1}}^{\dagger} \cdots B_{\lambda_{N}}^{\dagger}|0\rangle_{l+1} .
\end{aligned}
$$


Here the summation goes over all partitions $\lambda=\left(\lambda_{1}, \ldots, \lambda_{N}\right), \lambda_{1} \geq \lambda_{2} \geq \cdots \geq \lambda_{N} \geq 0$ of given weight $|\lambda|=\sum_{i} \lambda_{i}$ and lenght $l(\lambda)$. The coefficients in relations (3.3) are $z_{\lambda}=$ $\sum_{i} i^{m_{i}} m_{i}$ !, where $m_{i}$ is the number of $i$ 's in the partition $\lambda$, and $\varepsilon_{\lambda}=(-)^{|\lambda|-l(\lambda)}$. The same expression for the quasihole wave function in the Calogero model has been written down in ref. 18] and, also, the norm of this state has been given:

$$
{ }_{\nu}\left\langle 0\left|\chi(k) \chi^{\dagger}(k)\right| 0\right\rangle_{\nu}=\left(\begin{array}{c}
N \\
k
\end{array}\right) \prod_{i=0}^{k-1}[1+\nu i]
$$

The partition $\lambda=\left(\lambda_{1}, \ldots, \lambda_{N}\right)$ can be represented by the Young tableau with $N$ rows, each having $\lambda_{i}$ boxes. In the Calogero model, the quasiparticle $\pi^{\dagger}(n)$ is then represented by one row with $n$ boxes and quasihole $\chi^{\dagger}(n)$ by a single column with $N-n$ boxes.

In this representation it is evident that there is no fundamental distinction between quasiparticles and quasiholes for finite $N$, since they obviously represent the same type of excitations in two different bases. Using the properties of Schur function we can write a simple relation connecting these two bases:

$$
S_{n}=\sum_{l=1}^{n}(-)^{l+n} \sum_{i_{1}, \ldots, i_{l}=1}^{N} S_{1^{i_{1}}} \cdots S_{1^{i_{l}}}=\sum_{l=1}^{n}(-)^{l+n} \sum_{|\mu|=l}\left(\begin{array}{c}
\mu_{1}+\cdots+\mu_{N} \\
\mu_{1}, \ldots, \mu_{N}
\end{array}\right) S_{1}^{\mu_{1}} \cdots S_{1^{N}}^{\mu_{N}}
$$

and vice versa

$$
S_{1^{n}}=\sum_{l=1}^{n}(-)^{l+n} \sum_{i_{1}, \ldots, i_{l}=1}^{N} S_{i_{1}} \cdots S_{i_{l}}=\sum_{l=1}^{n}(-)^{l+n} \sum_{|\mu|=l}\left(\begin{array}{c}
\mu_{1}+\cdots+\mu_{N} \\
\mu_{1}, \ldots, \mu_{N}
\end{array}\right) S_{1}^{\mu_{1}} \cdots S_{N}^{\mu_{N}},
$$

where summations over $i_{\alpha}$ and $\mu$ are subject to condition $\sum_{\alpha=1}^{l} i_{\alpha}=\sum_{j=1}^{N} j \mu_{j}=n$. These relations hold for $\sigma_{\lambda}$ funtions also, i.e. we can apply these relations in both models.

\section{Outlook and discussion}

It is important to note that the whole picture is consistent only if the matrix $A$ is not diagonal. If we assume $A_{i j}=a_{i} \delta_{i j}$, then $\left[a_{i}, a_{j}^{\dagger}\right]=\delta_{i j}$, and that corresponds to the $l=-1$ tower which does not exist in the CS matrix model (although the $\nu=l+1=0$ case corresponds to $N$ bosons in the Calogero model). There is also another way to see this inconsistency. If we diagonalize the matrix $A$, the ground states factorize as $|0, l\rangle \sim\left(\prod \Psi_{i}^{\dagger}\right)^{l} \prod_{i<j}\left(a_{i}^{\dagger}-a_{j}^{\dagger}\right)^{l}$ and, generally, the vacuum conditions are not satisfied any longer, $\left(\sum_{i} a_{i}^{n}\right) \prod\left(a_{i}^{\dagger}-a_{j}^{\dagger}\right)^{l}|0\rangle_{\nu} \neq 0$ for $n \leq l$.

Similar arguments can be applied to the states of the form $\left(\sum_{i} a_{i}^{\dagger k}\right)^{n_{k}} \prod\left(a_{i}^{\dagger}-a_{j}^{\dagger}\right)^{l}|0\rangle_{\nu}$ with $\left[a_{i}, a_{j}^{\dagger}\right]=\delta_{i j}$, eqs. (11) and (12) in ref. [3], which correspond to the Laughlin states in a Fock space. For $l$ even, the above states are completely symmetric and can be written as $\left(\sum_{i} a_{i}^{\dagger k}\right)^{n_{k}}|0\rangle_{\nu}$, and for any odd $l$, the states are $\left(\sum_{i} a_{i}^{\dagger k}\right)^{n_{k}} \prod\left(a_{i}^{\dagger}-a_{j}^{\dagger}\right)|0\rangle_{\nu}$. Hence, all $l$-even states reduce to a Bose tower with $l=0$, and all $l$-odd states reduce to a Fermi tower with $l=1$. Moreover, the vacuum conditions are not satisfied. So, there is no one-to-one 
mapping between the CS matrix model states and the above Fock-space states. This leaves us with an unanswered question about the relation between $\mathrm{QH}$ physics and the finite CS matrix model, as was also observed in ref. 19].

Using the mapping from $\mathrm{F}_{\mathrm{ph}}^{\mathrm{CS}}(l)$ to $F_{\mathrm{symm}}^{\mathrm{Cal}}(l+1)$ we can show that there exists a mapping from the free Bose oscillators $\left\{b_{1}, b_{2}, \ldots, b_{N}, c_{0}\right\}$ to the observables in the CS matrix model $\left\{B_{1}, B_{2}, \ldots, B_{N}, C\right\}$, and vice versa [13]. This mapping offers a natural orthogonal basis in the physical Fock space $\left\{\prod\left(b_{k}^{\dagger}\right)^{n_{k}}|0, l\rangle\right\}$, an alternative to the orthogonal basis in terms of Jack polynomials proposed in ref. [6]

Also, the dynamical symmetry generators operating on degenerate states in a fixed tower of states $\mathrm{F}_{\mathrm{ph}}^{\mathrm{CS}}(l)$ are the same as those found in the Calogero model with $\nu=l+$ 1 [12, 13]. Moreover, one can introduce additional generators acting between different towers and so describe the larger dynamical symmetry operating on all degenerate states in the CS matrix model.

The relation between the Calogero model and the physics of anyons in the lowest Landau level has been described in ref. [8], and this relation is not restricted to the integer values of the interaction parameter in the Calogero model. It would be interesting to somehow enlarge this finite CS matrix model to describe also particles with fractional statistics. This can be easily done in the Fock-space approach.

We can define a new $\mathrm{SU}(N)$ invariant operator $D^{\dagger}=C^{\dagger 1 / q}$ such that $\mathcal{N}_{D}=q \mathcal{N}_{C}$, $\mathcal{N}_{D}$ and $\mathcal{N}_{C}$ being the number operators for $D$ 's and $C$ 's, respectively. The enlarged Fock space $F_{\text {phys }}^{\mathrm{CS} \text {,q }}$ is a space of all states of the type $\prod_{k}\left(B_{k}^{\dagger}\right)^{n_{k}} D^{\dagger l}|0\rangle_{\nu}$, for $l$ integer. From the action of the hamiltonian (2.6) on that Fock space we obtain $\nu=l / q+1$. Also, the generalized vacuum conditions (2.15) hold for any rational number $l \rightarrow l / q$, and therefore all identifications eqs. (2.16), 2.17) and (2.18) between the CS matrix and the Calogero model are true for any rational $\nu=l / q+1$. This construction is an allowed extension of Fock space for rational $\nu$, but we point out that the dynamical origin of such a picture is still missing.

In ref. [1] it was conjectured that the theory for QH fluid with the filling fraction $n / k$ would be level $k \mathrm{U}(n)$ non-commutative CS theory. In the string theory approach level $k$ $\mathrm{U}(n)$ non-commutative CS theory corresponds to the configuration of $n$ D2-branes and $2 k$ D8-branes in a background $B$-field [11]. Even in this approach, the dynamical description of the hierarchy structure and the mass gap of excitations in QH fluid is still missing.

In conclusion, we have discussed the Fock-space picture in detail and elucidated the $A$-representation of the CS matrix model. We have precisely formulated the connection between the states in the Fock spaces of the CS matrix and the Calogero model. We stress that although the models have similar Fock spaces and a common minimal algebraic structure, the complete algebraic structures are quite different. An important consequence of our analysis is that the operators $a_{i}$, elements of $S_{N}$-extended Heisenberg algebra, can be interpreted as elements belonging to the spectrum of the matrix $A$.

\section{Acknowledgments}

We would like to thank D. Svrtan for useful discussions. This work was supported by the Ministry of Science and Technology of the Republic of Croatia under contract No. 00980103. 


\section{A. Calogero model}

The hamiltonian of the (rational) Calogero model describes $N$ identical particles (bosons) interacting through an inverse square interaction subjected to a common confining harmonic force:

$$
H=-\frac{\hbar^{2}}{2 m} \sum_{i=1}^{N} \frac{\partial^{2}}{\partial x_{i}^{2}}+\frac{m \omega^{2}}{2} \sum_{i=1}^{N} x_{i}^{2}+\frac{\nu(\nu-1) \hbar^{2}}{2 m} \sum_{i \neq j}^{N} \frac{1}{\left(x_{i}-x_{j}\right)^{2}} .
$$

After performing a similarity transformation on the hamiltonian (A.1), we obtain the reduced hamiltonian acting on the space of symmetric functions $(m=\hbar=1)$ :

$$
H^{\prime}=\prod_{i<j}^{N}\left|x_{i}-x_{j}\right|^{\nu} H \prod_{i<j}^{N}\left|x_{i}-x_{j}\right|^{-\nu}=\sum_{i=1}^{N} a_{i}^{\dagger} a_{i}+E_{0}
$$

where the ground-state energy is $E_{0}=\omega N[1+(N-1) \nu] / 2$. Here, we have introduced the creation and annihilation operators

$$
\begin{aligned}
& a_{i}^{\dagger}=\frac{1}{\sqrt{2}}\left(-\partial_{i}-\nu \sum_{j, j \neq i}^{N} \frac{1}{x_{i}-x_{j}}\left(1-K_{i j}\right)+\omega x_{i}\right), \\
& a_{i}=\frac{1}{\sqrt{2}}\left(\partial_{i}+\nu \sum_{j, j \neq i}^{N} \frac{1}{x_{i}-x_{j}}\left(1-K_{i j}\right)+\omega x_{i}\right),
\end{aligned}
$$

satisfying the following commutation relations

$$
\left[a_{i}, a_{j}\right]=\left[a_{i}^{\dagger}, a_{j}^{\dagger}\right]=0, \quad\left[a_{i}, a_{j}^{\dagger}\right]=\left(1+\nu \sum_{k=1}^{N} K_{i k}\right) \delta_{i j}-\nu K_{i j} .
$$

The elementary generators $K_{i j}$ of the symmetry group $S_{N}$ exchange labels $i$ and $j$. The Fock space representation is defined by $a_{i}|0\rangle=0$ and $K_{i j}|0\rangle=|0\rangle$. The physical Fock space is defined by $S_{N}$-symmetric states $F_{\text {symm }}^{\mathrm{Cal}}=\left\{\prod_{n_{k}} B_{k}^{\dagger n_{k}}|0\rangle\right\}$, where $B_{k}=\sum_{i} a_{i}^{k}$ are

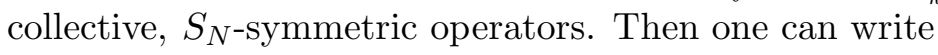

$$
H^{\prime} \prod_{n_{k}} B_{k}^{\dagger n_{k}}|0\rangle_{\nu}=\left[E_{0}+\omega \sum_{k=1}^{N} k n_{k}\right] \prod_{n_{k}} B_{k}^{\dagger n_{k}}|0\rangle_{\nu}
$$

The $S_{N}$-symmetric observables $B_{n}^{\dagger}$ creating the symmetric Fock space satisfy the following commutation relations:

$$
\begin{aligned}
& {\left[B_{m}, B_{n}\right]=\left[B_{m}^{\dagger}, B_{n}^{\dagger}\right]=0} \\
& {\left[B_{m}, B_{n}^{\dagger}\right]=n \sum_{r=0}^{m-1} \sum_{i=1}^{N} a_{i}^{r} a_{i}^{\dagger n-1} a_{i}^{m-r-1}=m \sum_{s=0}^{n-1} \sum_{i=1}^{N} a_{i}^{\dagger s} a_{i}^{m-1} a_{i}^{n-s-1} .}
\end{aligned}
$$

Including the observables of the type $B_{m, n}=\sum a_{i}^{\dagger m} a_{i}^{n}, m+n \leq N$ into consideration leads to the closed polynomial Lie algebra $\mathcal{B}_{N}^{\mathrm{Cal}}$ described in ref. [12]. 
As we have already mentioned, the same, universal relation (2.13) holds also in the

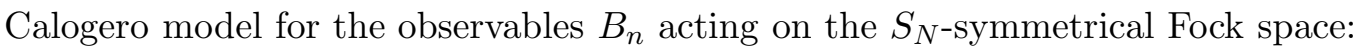

$$
\left[B_{i_{1}},\left[B_{i_{2}},\left[\ldots,\left[B_{i_{n}}, B_{n}^{\dagger}\right] \ldots\right]\right]=n ! \prod_{\alpha=1}^{n} i_{\alpha} B_{I-n},\right.
$$

where $I=\sum_{\alpha=1}^{n} i_{\alpha}$ and $i_{1}, \ldots, i_{n}, n=1,2, \ldots, N$. The representation of algebra (2.13) in $F_{\text {sym }}(\nu)$ is completely characterized by the minimal set of the generalized vacuum conditions:

$$
\begin{aligned}
B_{2} B_{2}^{\dagger}|0\rangle_{\nu} & =2 N(N \nu+1-\nu)|0\rangle_{\nu}, \\
B_{3} B_{3}^{\dagger}|0\rangle_{\nu} & =3 N\left[2(\nu-1)^{2}+\nu-3 N \nu(\nu-1)+N^{2} \nu^{2}\right]|0\rangle_{\nu}=y|0\rangle_{\nu}, \\
B_{3} B_{3}^{\dagger 2}|0\rangle_{\nu} & =54\left\{\nu B_{1}^{\dagger} B_{2}^{\dagger}+[N+(N-2)(\nu-1)+y / 27] B_{3}^{\dagger}\right\}|0\rangle_{\nu} .
\end{aligned}
$$

In the same way as in the CS matrix model one can show that all other generalized vacuum conditions can be calculated using the algebra (2.13) and relations (A.7). The action on $B_{n}$ on any state in the $S_{N}$-symmetric Fock space, and all matrix elements of the form ${ }_{\nu}\left\langle 0\left|\left(\prod B_{i}^{n_{i}}\right)\left(\prod B_{j}^{\dagger n_{j}}\right)\right| 0\right\rangle_{\nu}$ are uniquely determined by (2.13) and (A.7).

\section{References}

[1] L. Susskind, The quantum hall fluid and non-commutative Chern-Simons theory, hep-th/0101029.

[2] A.P. Polychronakos, Quantum hall states as matrix Chern-Simons theory, J. High Energy Phys. 04 (2001) 011 hep-th/0103013.

[3] S. Hellerman and M. Van Raamsdonk, Quantum hall physics equals noncommutative field theory, J. High Energy Phys. 10 (2001) 039 hep-th/0103179.

[4] D. Karabali and B. Sakita, Chern-Simons matrix model: coherent states and relation to Laughlin wavefunctions, hep-th/0106016.

[5] F. Calogero, Solution of a three body problem in one-dimension, J. Math. Phys. 10 (1971) 2191; Ground state of one-dimensional N body system, J. Math. Phys. 10 (1971) 2197;

Solution of the one-dimensional $N$ body problems with quadratic and/or inversely quadratic pair potentials, J. Math. Phys. 12 (1971) 419.

[6] D. Karabali and B. Sakita, Orthogonal basis for the energy eigenfunctions of the Chern-Simons matrix model, hep-th/0107168.

[7] T.H. Hansson, J.M. Leinaas and J. Myrheim, Dimensional reduction in anyon systems, Nucl. Phys. B 384 (1992) 559.

[8] L. Brink, T.H. Hansson, S. Konstein and M.A. Vasiliev, The Calogero model: anyonic representation, fermionic extension and supersymmetry, Nucl. Phys. B 401 (1993) 591 hep-th/9302023.

[9] S. Iso and S.J. Rey, Collective field theory of the fractional quantum Hall edge state and the Calogero-Sutherland model, Phys. Lett. B 352 (1995) 111 hep-th/9406192.

[10] A.P. Polychronakos, Integrable systems from gauged matrix models, Phys. Lett. B 266 (1991) 29 . 
[11] O. Bergman, Y. Okawa and J.H. Brodie, The stringy quantum Hall fluid, J. High Energy Phys. 11 (2001) 019 hep-th/0107178.

[12] L. Jonke and S. Meljanac, Dynamical symmetry algebra of the Calogero model, Phys. Lett. B 511 (2001) 276 hep-th/0105043.

[13] L. Jonke and S. Meljanac, Bosonic realization of algebras in the Calogero model, Phys. Lett. B 526 (2002) 149 hep-th/0106135.

[14] S.B. Isakov and J.M. Leinaas, Algebra of one-particle operators for the Calogero model, Nucl. Phys. B 463 (1996) 194 hep-th/9510184;

J.M. Leinaas, Generalized statistics and the algebra of observables, hep-th/9611167;

S.B. Isakov, J.M. Leinaas, J. Myrheim, A.P. Polychronakos and R. Varnhagen, Algebra of observables for identical particles in one dimension, Phys. Lett. B 430 (1998) 151 hep-th/9702066.

[15] A.J. Macfarlane and H. Pfeiffer, On characteristic equations, trace identities and Casimir operators of simple Lie algebras, J. Math. Phys. 41 (2000) 3192;

L. Jonke and S. Meljanac, Algebraic description of $S_{N}$-symmetric states in the Calogero model, IRB-TH-06/01.

[16] Y. Ohnuki and S. Kamefuchi, Quantum field theory and parastatistics, Tokyo University Press 1982.

[17] I.G. Macdonald, Symmetric functions and Hall polynomials, 2nd edition, Claredon Press, Oxford 1998.

[18] A.P. Polychronakos, Quasihole wave functions for the Calogero model, Mod. Phys. Lett. A 11 (1996) 1273.

[19] A.P. Polychronakos, Quantum hall states on the cylinder as unitary matrix Chern-Simons theory, J. High Energy Phys. 06 (2001) 070 hep-th/0106011. 